\title{
MORE INEQUALITIES FOR POSITIVE LINEAR MAPS
}

\author{
R. SHARMA AND A. THAKUR
}

Abstract. We derive inequalities for the norm of the variance of matrices. It is shown that unital linear maps on $2 \times 2$ matrices preserve the commutativity properties of matrices. This feature allows us to generalize several inequalities for such maps. We show by way of an example that this technique cannot be extended to the case of $n \times n, n \geqslant 3$, matrices.

Mathematics subject classification (2010): 47A3, 15A 60.

Keywords and phrases: linear maps, variance, Chebyshev's inequality, Schwarz's inequality.

\section{REFERENCES}

[1] R. Bhatia, Positive Definite Matrices, Princeton University Press, 2007.

[2] R.V. KADISON, A generalized Schwarz inequality and algebraic invariants for operator algebras, Ann. Math. 56 (1952), 494-503.

[3] R. Bhatia, C. Davis, A better bound on the variance, Amer. Math. Monthly 107 (2000), 353-357.

[4] M. D. CHOI, A Schwarz, inequality for positive linear maps on $C^{*}$-algebras, Illinois J. Math. 18 (1974), $565-574$.

[5] R. Bhatia, R. Sharma, Some inequalities for positive linear maps, Linear Algebra Appl. 436 (2012), $1562-1571$.

[6] J.C. Bourin, E. RiCARD, An asymmetric Kadision's inequality, Linear Algebra Appl. 433 (2010), 499-510.

[7] R. Bhatia, Matrix Analysis, Springer-Verlag, 1997.

[8] R. Bhatia And F. KitTAneh, The matrix arithmetic-geometric mean inequality revisited, Linear Algebra Appl. 428 (2008), 2177-2191. 